\title{
Data Mining of Internet of Things under the Cloud Computing Platform
}

\author{
Xiaoli Liu \\ Guangzhou Huali College of Science and Technology China Guangzhou 511325
}

Keywords: Cloud computing platform; Internet of things; Data mining; Application

\begin{abstract}
With the rapid development of information technology, as a key technology in the information era of the Internet of things has been widely used in various industries, and based on the cloud computing platform on the Internet of things to explore is of great significance for the application of Internet of things in the industry in the current. Based on the characteristics of Internet of things and cloud computing, this paper studies and analyzes data mining of Internet of things under cloud computing platform with a view to provide theoretical support for the application of Internet of things technology under cloud computing platform.

With the rapid development of Internet technology, cloud computing and Internet of things technology are widely applied to all aspects of people's work and life. As a decisive factor in the current competition among enterprises in various fields, data mining has also become the main content of Internet of things applications. In order to deal with the various industries for networking applications in data mining, this paper focuses on the research of mining and discusses the calculation of IOT data under the background of cloud based data to solve the problem of cloud computing platform networking mining problems providing support and help for networking applications in various industries.
\end{abstract}

\section{Part One Internet of Things and Cloud Computing}

\section{Internet of Things}

The Internet of things is that consists of a large number of small sensors and the exchange of information and communication network technology, which consists mainly of small sensors which are closely related to our life and work of the large server, super computer, computer, mobile phone and other portable network equipment. The main features are as follows: (1) the Internet is based on Internet technology which is the extension and expansion of the Internet; (2) networking client extended to any item as long as the establishment of information exchange and communication to set up networking; (3) The network can realize the protection of the privacy of the user terminal, and through the Internet to find a solution for each event. With the rapid development of computer technology and Internet technology, IOT technology is widely applied to various kinds of network information exchange and integration because of its intelligent perception, recognition technology and various mobile terminals. Therefore, the Internet of things is known as the core of the future development of network communication technology. As the practical application of Internet technology, the Internet of things mainly realizes the various businesses and jobs that users use Internet technology to carry out.

\section{Cloud Computing}

Cloud computing is the effective utilization of virtual resources in the network by means of computer hardware and software, development platform and computer protocol, and rationally configuring these resources according to different loads, so as to raise the utilization ratio of virtual resources. The main features of cloud computing are as follows: (1) Service. The significance of cloud computing is to provide services for users, which means users can get the corresponding services without needing to solve the specific working mechanism of cloud computing. (2) Supply reliability. Cloud computing is a system composed of a large number of servers and computer groups to provide users with data processing and computing service, in order to reduce the failure probability calculation due to the increase in the number of computers, cloud computing systems often through the use of software in data redundancy and distributed storage to improve the reliability of the data; (3) High efficiency. Cloud computing is able to provide efficient services for 
customers because it integrates a lot of computer storage space and computing power; (4) Economy. Because cloud computing is a way of clustering business computers to improve the efficiency of computers, this way costs less than buying or using large computers. (5) Service diversity. Users can get the corresponding services according to their needs and the corresponding expenses, which not only provides users with more choices, but also saves users a certain cost.

\section{Key Problems to be Solved in the Data Mining of the Internet of Things}

The study found that to achieve data mining problem under cloud computing platform, we must discuss and study the key issues of data mining in Internet of things, and the key problems are as follows.

Traditional Patterns are Difficult to Apply. The central mode is the main mode of data mining, because it focuses on the relative concentration of data, and Internet of things data are stored in different locations, so the effect of Internet of things is not obvious in this mode.

High Requirements for Central Node Hardware. Because the Internet of things itself has the problems of large data processing and many sensor nodes, in order to meet the characteristics of the Internet of things, we must provide high-performance central nodes for practical applications.

Limited Node Resource. Due to the relatively few node resources in the Internet of things, the distributed nodes in the Internet of things must undertake the pre-processing and delivery of raw data in practical applications.

Influence of External Factors. Based on user data security and privacy considerations, IOT can not place all data in the same data storage space in practical applications, which greatly increases the difficulty of data mining by the Internet of things. Therefore, the existing Internet of things technology and hard and soft software can not provide strong support for its data mining.

\section{Part Two Data Mining of Internet of Things Based on Cloud Computing}

This section is mainly the cloud computing platform for mining process platform of IOT based on data and data integration, for example in networking, perception layer, transport layer, data layer and service layer data mining as part of the Internet of things which is constructed and platform module establishment, methods and ideas to construct the module will be launched in the following.

\section{IOT Perception Layer}

The perception layer of IOT is mainly the relevant data that were collected by the arrangement of acquisition nodes in the target area, the acquisition node is mainly composed of cameras, sensors and other instruments, and through the acquisition, the collected data is aggregated by certain means to each node, then these data through the summary before and after treatment, we can build the cloud based data processing platform is transferred to the transport layer of the late.

\section{Transport Layer}

The transport layer is mainly the realization of data transport function, and network data transmission layer of cloud computing platform should have the characteristics of reliability, high efficiency and so on, in the practical application, the platform should be combined with the sensor, cable networks, wireless networks and other data transmission to achieve the construction of data transmission network networking data mining based on cloud computing technology the construction, and transport layer can be achieved on the perception layer acquisition efficient data transfer to a certain extent, and achieve higher efficiency and level of information transfer and contact, and the establishment of the transport layer can also transmit data monitoring system to Internet data mining system.

\section{Data Layer}

Because the Internet data mining has a large amount of data and data types and other characteristics, which leads to a network of cloud computing data mining based on platform construction which has very high requirements for networking data storage devices and data processing performance, and for the networking of data mining system, the data layer is mainly composed of a data source conversion module and distributed storage module, the data source conversion module mainly on the Internet of things in different types of data conversion, and the distributed storage module is mainly the realization of data storage networking inside. 
In the different objects within the Internet often need to be represented by different data types, which leads to heterogeneous data within the Internet that has become the fundamental characteristics of the data, but in actual life, the study object that is more conducive to the use of data using different types of data, puts forward high requirements these characteristics of converter to the data source within the internet. Analysis of data networking based on constructing the mining system that has an important effect on the feasibility of data integrity, data source converter for data storage networking in the mining process, and networking of data mining and data decoding in distributed data packet system also needs a data source converter support, so in the IOT data mining system, each NameNode node file type for the PML file is mainly due to the PML file to object description in a special way, and the PML can of goods and articles with information and exchange of information that plays an irreplaceable role. For example: the cloud based networking data mining system, the PML file for the data acquisition, transmission, storage and other functions have played an important role, and the goods by the modeling of information collected by the location, environment, history and use function of information can be accurately described for the goods, it also has very important influence for the networking of data mining process and quality.

\section{Data Mining Service Layer}

Data mining service layer is an important part of data mining system of IOT, which is mainly divided into the following parts: data preparation module, data mining engine module and user module. Its specific contents will be discussed and studied in detail in this section.

Data Preparation Module. The data preparation module is mainly to collate, convert and clean up the data collected from the Internet of things.

Data Mining Engine Module. Data mining engine module is the main function through the data mining algorithm and pattern evaluation module for network data mining system provides complete data service, data division, correlation analysis, aggregation and trend and deviation analysis, and achieving these functions is the base and core of using and processing algorithms and the improvement of construction and calculation the platform of cloud data mining algorithm with high efficiency.

User Module. The user module is the most important part in data mining in the Internet of things, the first is to realize the visualization of functional areas, and system designers should also consider the efficiency of this system that is friendly and the operation process of data mining, in order to facilitate the user to carry on the operation and use. But, in the actual use of the process, data processing and storage needs to be realized through some external devices, so the data mining service layer design, which often needs the service layer module for open interface reservation and design in order to enrich the experience of the operating system user data mining.

\section{Conclusion}

To sum up, the construction of the IOT data mining system based on cloud computing platform has an extremely important significance and role for people's life and work. Based on the characteristics of cloud computing and the Internet of things, this paper analyzes and studies the construction process of data mining system of IOT, in order to provide theoretical support and practical reference for the construction of data mining system of Internet of things under the background of cloud computing.

\section{Reference}

[1] Huaiguo Li, Xiaole Yao. Large data and cloud computing platform and application[J].New Communication in China, 2017(2): 122.

[2] Shenghe Wu. Key technology of multimedia cloud computing platform[J].Electronic Technology and Software Engineering, 2017(17): 174.

[3] Zhangguang $\mathrm{Wu}$, Baojian Chen, Weimin $\mathrm{Wu}$. Data mining based on Internet of things[J].Science and Wealth, 2017(30): 98-103. 